Editorial

\title{
Monográfico sobre factores psicosociales. Salud laboral y salud mental: estado de la cuestión
}

\author{
Monograph on psychosocial factors. Occupational health and mental health: \\ state of the art
}

\author{
Macarena Gálvez Herrer ', Jerónimo Maqueda Blasco² , José Carlos Mingote Adán ${ }^{1}$ \\ 1. Programa de Atención Integral al Profesional Sanitario Enfermo (UVOPSE-PAIPSE) Consejería de Sanidad. \\ Comunidad Autónoma de Madrid. España. \\ 2. Escuela Nacional de Medicina del Trabajo. Instituto de Salud Carlos III. Madrid. España.
}

Recibido: 29-09-11

Aceptado: 30-09-11

\section{Correspondencia}

Macarena Gálvez Herrer.

UVOPSE-PAIPSE

Pabellón 8, Ciudad Universitaria

28040 Madrid

Tfno: 913303926

e-mail: mgalvez.hcsc@salud.madrid.org

Resumen

Se presenta una nota informativa sobre el próximo número monográfico de la revista Medicina $y$ Seguridad en el Trabajo. Se incluye el fundamento teorico-práctico que ha impulsado su elaboración y el contenido principal del mismo.

Desde una perspectiva multidisciplinar, se abordarán temas tales como el acoso laboral, la violencia externa en el lugar de trabajo, los procesos de estrés laboral y desgaste profesional, la perspectiva de género en la prevención de riesgos laborales, el conflicto trabajo-familia, la salud mental y laboral en población trabajadora inmigrante, los problemas de drogodependencias y alcohol en el trabajo, la situación de los trabajadores con discapacidad, de los trabajadores con trastorno mental, los modelos de bienestar y salud aplicados al trabajo y la gestión de la salud mental como parte de la salud laboral en las organizaciones.

Med Segur Trab (Internet) 2011; 57 (224) 188-189

Palabras clave: Salud mental, salud laboral, factores psicosociales.

Abstract

It presents a brief about the next special issue of the journal Medicina $y$ Seguridad en el Trabajo. It includes theoretical and practical foundation that has driven its development and the main content of it.

From a multidisciplinary perspective, issues such as bullying, external violence in the workplace, the processes of job stress and burnout, gender in risk prevention, work-family conflict, mental health and occupational health in migrant worker, drug addiction and alcoholism in the workplace, the situation of workers with disabilities, workers with mental disorders, the welfare models applied to occupational health and management mental health as part of occupational health.

Med Segur Trab (Internet) 2011; 57 (224) 188-189

Keywords: Mental bealth, occupational health, psychosocial factors. 
Para cerrar el año 2011 la Escuela Nacional de Medicina del Trabajo, Instituto de Salud Carlos III, en colaboración con el Programa de Atención Integral al Profesional Sanitario Enfermo de la Comunidad de Madrid (UVOPSE-PAIPSE) han proyectado la elaboración de un monográfico de la revista Medicina $y$ Seguridad en el Trabajo en el que compilarán artículos de revisión sobre los riesgos psicosociales emergentes y su estrecha relación con la salud mental.

Según la Agencia Europea para la Seguridad y la Salud en el Trabajo ${ }^{1}$, en la actualidad son cinco las áreas del ámbito laboral que se relacionan con los riesgos psicosociales emergentes. Éstas áreas son las nuevas formas de contratación laboral e inseguridad en el puesto de trabajo, el envejecimiento de la población activa, la intensificación del trabajo, las fuertes exigencias emocionales en el mismo y el desequilibrio entre la vida laboral y personal. A estas situaciones se unen cambios en la población activa de la Unión Europea como son la feminización de la población trabajadora y la incorporación de trabajadores inmigrantes.

Algunos estudios, como el metaanálisis de Woo y Postolache ${ }^{2}$, realizado sobre las publicaciones relativas a la relación entre trabajo y salud mental entre los años 1966 a 2007, plantean que los problemas de salud mental pueden revertirse o prevenirse si se actúa sobre las características del trabajo y que los factores del trabajo que se relacionan con los problemas de salud mental son tanto de tipo físico, biológico, químico, social, psicológico, como oganizacional.

Por todo ello, el Observatorio de Riesgos de la Agencia Europea 3 propone algunas prioridades en el estudio y la investigación de los riesgos psicosociales en el trabajo:

- Fomento de entornos de trabajo positivos, claves para la prevención

- Estudio de los riesgos psicosociales asociados a los cambios organizativos y sociales

- Interacción entre factores de riesgo psicosocial y problemas de salud (como los trastornos musculoesqueléticos)

- Ergonomía física y cognitiva y su repercusión en el estrés laboral.

Considerando estos aspectos de actualidad científica y necesidades detectadas por organismos oficiales, el monográfico pretende aportar una revisión actualizada de los factores psicosociales del trabajo que se relacionan con posibles riesgos psicosociales y alteraciones sobre la salud mental.

Diversos expertos en la materia, desde una perspectiva multidisciplinar, abordarán temas tales como el acoso laboral, la violencia interna y externa en el lugar de trabajo, los procesos de estrés laboral y desgaste profesional, la perspectiva de género en la prevención de riesgos laborales, la conciliación trabajo-vida extralaboral, la salud mental y laboral en población trabajadora inmigrante, los problemas de drogodependencias y alcohol en el trabajo, la situación de los trabajadores con discapacidad, de los trabajadores con trastorno mental, los modelos de bienestar y salud aplicados al trabajo y la gestión de la salud mental como parte de la salud laboral en las organizaciones. El abordaje de estos temas incluirá tanto una actualización teórica como una visión aplicada y esperamos por tanto sea de utilidad a los lectores de esta revista.

\section{REFERENCIAS BIBLIOGRÁFICAS}

1. Agencia Europea para la Seguridad y la Salud en el Trabajo. Previsiones de los expertos sobre la aparición de riesgos psicosociales en relación con la seguridad y la salud en el trabajo. FACTS 74. Disponible en: http://osha.europa.eu/es/ publications/factsheets/ 74 .

2. Woo, J.M. y Postolache, T.T. The impact of work environment on mood disorders and suicide: evidence and implications. Int Disabil Hum Dev 2008:7: 185-200.

3. Agencia Europea para la Seguridad y la Salud en el Trabajo. Observatorio de Riesgos. Riesgos Nuevos y Emergentes para la Seguridad y Salud en el Trabajo. Luxemburgo: Oficina de publicaciones oficiales de las Comunidades Europeas, 2009. 